\title{
Si può parlare di Dio nel contesto della scienza contemporanea?
}

\section{Is it possible to speak about God in the context of contemporary science?}

\author{
GIUSEPPE TANZELLA-NITTI \\ Facoltà di Teologia \\ Scuola Internazionale Superiore per la Ricerca Interdisciplinare - SISRI \\ Pontificia Università della Santa Croce, Roma \\ tanzella@pusc.it
}

Riassunto. La questione di Dio, trascurata nella filosofia del Novecento, riemerge inaspettatamente nella scienza contemporanea. Tuttavia, l'accesso alla nozione di Dio tramite la razionalità scientifica non implica una dimostrazione della sua esistenza, ma piuttosto un'interrogazione sulla sua significatività in contesto scientifico: è necessario che esista uno spazio disponibile per un discorso sul Logos che la razionalità scientifica possa riconoscere. Si segnalano qui come candidate quattro aree semantiche contrassegnate da: (i) il carattere incompiuto del linguaggio formale; (ii) il carattere ontologicamente incompiuto della realtà fisico-contingente; (iii) la razionalità, ordine ed intelligibilità del cosmos; (iv) la dimensione personalista-esistenziale dell'attività scientifica. La domanda sul senso e l'apertura all'indicibile nascono all'interno dell'analisi del conoscere scientifico, e non fuori di esso, poiché il metodo e gli strumenti del lavoro scientifico possono essere trascesi senza essere contraddetti.

Parole chiave: Scienza e fede; Discorso su Dio; Filosofia della scienza.

Abstract. The question of God, neglected in the philosophy of the twentieth century, emerges unexpectedly in contemporary science. However, the access to the notion of God through scientific rationality does not imply a demonstration of its existence, 
but rather a question on his significance within such context: there must be a space available for a discourse about the Logos which the scientific rationality can recognize. This space can be instantiated in four semantic areas marked by: (1) the incomplete nature of the formal language; (2) the incomplete nature of the ontologically contingent physical reality; (3) the rationality, order and intelligibility of the cosmos; (4) the personalist-existential dimension of scientific activity. The question about meaning and the opening to the "ineffable" arise within the analysis of scientific knowledge because the method and tools of scientific work can be transcended without being contradicted.

Keywords: science and religion; discourse about God; philosophy of science.

Un semplice sguardo agli scaffali di divulgazione scientifica in una libreria delle nostre città ci mostrerebbe oggi che non pochi libri hanno in copertina la parola "Dio". Nei volumi di lingua inglese, nell'Indice dei Concetti, il termine God si è conquistato ormai uno spazio fra General Relativity e Grand Unified Theory. Sebbene parte di questo fenomeno, come già riconosciuto da alcuni autori, è dovuto al fatto che le Big Questions attraggano ancora oggi il pubblico - la domanda se l'universo abbia un Creatore e se la vita abbia un'origine in Dio sono, appunto, esempi di queste grandi domande - resta significativo che la nozione di Dio divenga oggetto di dibattito in un ambito, la scienza, dove non ce lo aspetteremmo. Eppure già Albert Einstein anni addietro ci aveva avvertito di non essere interessato a un fenomeno particolare o al significato di una determinata riga spettrale: ciò che attraeva era sapere come Dio aveva creato il mondo. Alcuni pensatori hanno notato che la "questione su Dio", trascurata nella filosofia del Novecento, perché ritenuta una domanda troppo impegnativa in un clima di pensiero debole e relativista come quello che stiamo vivendo, riemerge inaspettatamente nella scienza. Il fenomeno, però, va ben compreso, anche con la sua ambiguità: alcuni scienziati (o i divulgatori delle loro scoperte) sono infatti persuasi, come Paul Davies, che la scienza ci offre oggi vie verso Dio più solide di quelle offerte dalle religioni, mentre altri, come Stephen Hawking, sono convinti che la scienza contemporanea può facilmente mostrare che l'universo non ha bisogno di alcun Creatore. Come mettere ordine in queste diverse prospettive? 
Può valer la pena anche ricordare quali sono i campi della ricerca scientifica, o quali riflessioni svolte a partire da essa, fanno oggi riferimento alla nozione di Dio, o a nozioni equivalenti (Creatore, Assoluto, Intelligenza ordinatrice, ecc.) per affermarla o per negarla. Tale nozione pare emergere in tre ambiti principali: a) il problema dell'origine del reale fisico, soprattutto in cosmologia; b) la domanda sull'intelligibilità della natura e l'origine delle leggi naturali, e dunque in rapporto con la matematica e le scienze naturali; c) il dibattito circa la possibilità delle scienze di affermare o negare l'esistenza di una finalità in natura, come accade in biologia a proposito del dibattito sui “motori” dell'evoluzione o sull'intelligent design, o ancora in cosmologia, quando si espongono le tesi del Principio antropico.

In questa Lecture $^{1}$ cercheremo di esaminare più da vicino i motivi filosofici del perché il tema di Dio torna ad essere dibattuto nel contesto scientifico, chiedendoci se, in questo contesto, tale nozione abbia un significato, oppure non lo possegga affatto. Un autore come Mariano Artigas fu particolarmente sensibile, nei suoi scritti, al tema che qui ci occupa e desideriamo trattarlo cominciando come avrebbe fatto lui, ovvero fornendo subito dei chiarimenti di carattere epistemologico.

\section{Alcuni chiarimenti epistemologici}

In linea generale, pare chiaro che una nozione di Dio, come questa viene abitualmente compresa in sede filosofica (oppure teologica) non possa essere oggetto delle scienze naturali, le quali esaminano il reale nei suoi aspetti sperimentabili, soprattutto quantitativi.

La filosofia introduce l'accesso alla nozione Dio, fino a formulare possibili conclusioni circa la sua esistenza o meno, partendo sia dal cosmo (teologia naturale, metafisica), sia dall'uomo (questioni di ambito esistenziale: libertà, senso della vita, coscienza morale, ecc.). L'immagine di Dio cui giunge la razionalità filosofica possiede attributi che non esauriscono, da soli, l'immagine di Dio come questa viene compresa dalla riflessione

Il testo corrisponde alla III Mariano Artigas Memorial Lecture, la cui prolusione è stata fatta dall'autore a Pamplona, il 20 ottobre di 2015. 
teologica a partire dalla rivelazione biblica consegnata dalla tradizione ebraico-cristiana. Tuttavia, per poter essere significativa per la ragione umana, l'immagine di Dio della rivelazione biblica deve anche soddisfare eventualmente superare, ma non contraddire - gli attributi di un'immagine di Dio colta dalla ragione filosofica.

Interrogarsi circa "l'accesso alla nozione di Dio attraverso la razionalità scientifico-filosofica" non significa occuparsi di alcuna dimostrazione dell'esistenza di Dio nel contesto della razionalità delle scienze, in quanto ciò sarebbe contraddittorio a motivo dell'oggetto proprio dell'analisi scientifica, che non può avere Dio come oggetto adeguato; equivale invece a chiedersi se la nozione di Dio sia una nozione significativa per un soggetto il cui contesto di pensiero sia quello della razionalità scientifica.

Rispondere a questa domanda non equivale a mostrare la pertinenza o meno di un riferimento a Dio negli ambiti di discussione scientifica prima enunciati, o in altri analoghi. Si tratta invece di mostrare se l'uomo di scienza è tenuto a prendere in considerazione il sapere filosofico su Dio e/o a prestare ascolto alla Rivelazione e alla teologia che gli parlano di Dio, perché egli riconosce, anche nel contesto della razionalità scientifica, che tale nozione non sia un "nonsenso".

Nelle celebri "cinque vie" formulate per mostrare l'esistenza di Dio (Summa theologiae, I, q. 2, a. 3), Tommaso d'Aquino (1224-1274) divideva ogni prova in due parti: a) la presentazione di un ragionamento filosofico che giunge ad un Primo motore in Atto, ad una Prima Causa efficiente, ad un Essere necessario per sé, alla causa infinita delle partecipazioni/perfezioni finite, ad una Causa finale; b) l'affermazione «... e questo tutti chiamano Dio» (intelligunt, nominant, dicunt Deum). Si tratta adesso di provare se la seconda parte dell'articolazione, che rimanda ad una precomprensione (euristica, esistenziale, intellettuale, razionale, ecc.) del termine Dio, può essere riconosciuta anche da coloro il cui approccio al reale è modellato dalla razionalità delle scienze.

Affinché una nozione di Dio di ambito filosofico o teologico non venga giudicata un "non senso" - cioè, sia giudicata sensata - da una razionalità di ambito scientifico, occorre che esista una "area semantica" disponibile per 
un discorso (Logos) sull'Assoluto, area che la razionalità scientifica possa riconoscere, ovvero indicare, come significativa. Si segnalano come "candidate", a proposito di tale riconoscimento, le seguenti quattro aree semantiche:

a) Area cui additta il carattere incompiuto del linguaggio formale (apertura ad una trascendenza oltre il linguaggio)

b) Area cui additta il carattere ontologicamente incompiuto della realtà fisico-contingente (apertura verso un fondamento metafisico-necessario, oltre il plano empirico)

c) Area di senso che dia ragione della razionalità, ordine ed intelligibilità del cosmos (oltre la natura del cosmos stesso: percezione di un Logos ut ratio)

d) Area di senso che dia ragione della dimensione personalista-esistenziale dell'attività scientifica (apertura verso il senso ultimo della ricerca della verità: percezione di un Logos ut verbum).

In particolare, queste quattro aree sono in certo modo collegate ai "fondamenti filosofici” del conoscere scientifico. Si tratta, rispettivamente, di fondamenti: a) logico-epistemologici; b) ontologici; c) logico-razionali; d) antropologici. Si tratta di "aperture" del linguaggio e del metodo scientifico verso altre forme di conoscenza e altri saperi. Potremmo parlare di 4 "finestre" che sono presenti nella casa della scienza. Le finestre appartengono alla casa, cioè il metodo scientifico può tematizzarle, segnalarle, ma attraverso di esse si vede "più in là del metodo scientifico" in senso stretto. Attraverso di esse possiamo accedere al mondo esterno, vediamo gli ampi panorami che circondano la casa della scienza.

In questo approccio seguiremo e svilupperemo una intuizione che don Mariano Artigas lasciò chiara nella sua opera The Mind of the Universe (Artigas 2000), quando affermava che la ciencia "si trascende se stessa". In quest'opera l'autore segnala 3 "presupposti” della scienza, la cui analisi però appartiene alla filosofia ed alla teologia: presupposti ontologici, presupposti epistemologici, presupposti etici. «Existen tres tipos de presupuestos, afirmaba. El primero (ontológico) se refiere a la inteligibilidad y a la racionalidad de la naturaleza y está ligado al orden natural. El segundo (epistemológico) se refiere a la capacidad humana de conocer el orden na- 
tural e incluye las diferentes formas del razonamiento científico. El tercero (ético) se refiere a los valores que la actividad de la ciencia implica: incluye la búsqueda de la verdad, el rigor, la objetividad, la humildad intelectual, la cooperación» (Artigas 2000, xix)

\section{Il senso di un riferimento all'Assoluto oltre il linguaggio formale}

Come sappiamo, il programma del neopositivismo logico (R. Carnap, B. Russell, O. Neurath), intendeva poter giungere, in sede logica, a linguaggi assiomatici non ambigui e formalmente completi. Una volta riconosciuto alla logica il valore di teoria "fondativa”, tale proprietà veniva automaticamente riconosciuta anche alla matematica, fornendo così una base completa a tutte le scienze matematizzabili, cioè al sapere empirico tipico delle scienze naturali. In tal modo, gli asserti di carattere metafisico (o meta-empirico) sarebbero stati smascherati come privi di qualsiasi significato, perché senza alcuna corrispondenza con il mondo dei fatti.

Tale programma si rivelò impraticabile, perché si scontrava con l'impossibilità di definire, in forma assiomatica, un sistema logico-matematico formalmente completo, che fornisse cioè dal suo interno tutti gli elementi necessari per formulare ogni decisione e realizzare ogni calcolo. Inoltre, esso si imbatteva anche con la necessità di inglobare ogni sistema e ogni linguaggio, al fine di renderli comprensibili, all'interno di un meta-linguaggio o di un meta-sistema di valore più generale, esterno a quello di partenza: il linguaggio oggetto (linguaggio "di cui” si parla) deve necessariamente distinguersi dal meta-linguaggio (linguaggio “in cui” si parla).

Non operare le necessarie generalizzazioni verso progressivi e sempre aperti livelli di linguaggio conduce necessariamente a delle antinomie, espressione, a loro volta, dell'irriducibilità del rapporto fra "semantica" e "sintassi". Ovvero: a) non esiste un sistema sintattico completo (regole da seguire) che possa prescindere da una semantica (significato da dare agli oggetti che seguono le regole); b) se il sistema risulta completo dal punto di vista della sintassi, la semantica va cercata su basi esterne al sistema stesso. 
Una delle principali obiezioni mosse dal neopositivismo logico alla metafisica e al linguaggio religioso è che ogni discorso su ciò che trascende il livello empirico (come appunto un discorso su Dio) non possiede i caratteri di un linguaggio universale, non ambiguo e comunicabile, perché riguarderebbe asserti non falsificabili, in quanto slegati dal "mondo dei fatti".

Per fornire una risposta a questa critica, risulta assai utile ricordare l'itinerario filosofico di Ludwig Wittgenstein (1889-1951). Impegnatosi a fondare un linguaggio che potesse eliminare dal discorso filosofico ogni ambiguità e nonsenso, proprio mediante un rigido collegamento con il mondo dei fatti, Wittgenstein finì col mostrare implicitamente che tale programma era impraticabile, allontanandosi così dal circolo di Vienna e dalla prospettiva neopositivista.

A differenza di quanto affermavano i neopositivisti, nella riflessione di Wittgenstein la trascendenza e l'esistenza di un Assoluto non venivano negate, bensì colte all'interno della filosofia come apertura, come un rimando all'indicibile, a un senso e a un fondamento esterni al linguaggio filosofico, senza dei quali tale linguaggio sarebbe stato impossibile (il "primo" Wittgenstein del Tractatus); ma esse vengono colte anche all'interno della vita vissuta, come ricerca delle ragioni per vivere e come criterio di verità del significato degli stessi termini del linguaggio (il "secondo" Wittgenstein).

Per Wittgenstein non è possibile negare il problema del senso, ma il fatto che sia inesprimibile «all'interno del mondo dei fatti» ne fa, in termini scientifici, uno pseudo-problema; solo se fosse possibile prendere prospettiva dal mondo logico dei fatti delle scienze ci accorgeremmo di esso, e l'accorgercene possiamo indicarlo come «qualcosa di mistico». Così dal Tractatus Logico-philosophicus (1922):

Il senso del mondo deve essere fuori di esso (6.41).

Noi sentiamo che, anche se tutti i possibili interrogativi delle scienze ricevessero una risposta, i nostri problemi della vita non ne sarebbero neppure sfiorati (5.52).

C’è davvero l'inesprimibile. Esso si mostra, è "il mistico" (6.522)

Il mistico non è come è il mondo, ma che il mondo è [esiste] (6.44). 
Il percorso filosofico di Wittgenstein supera sia la posizione kantiana sia quella neopositivista. La domanda sul senso e l'apertura all'indicibile nascono all'interno dell'analisi del conoscere scientifico, e non fuori di esso, come sostenuto da Kant, sebbene non possa essere adeguatamente espressa; il meta-linguaggio nasce come esigenza dei limiti del linguaggio riconosciuti all'interno del linguaggio medesimo. L'impiego dell'analisi del linguaggio per un discorso critico sulla nozione di Dio incontra simultaneamente sia i limiti, sia la trascendenza della nostra conoscenza: l'uomo è più del suo linguaggio e l'analisi del linguaggio, nel toccare i limiti della razionalità umana, ne rivela l'ineffabilità. Wittgenstein conclude la parabola dell'empirismo e del neopositivismo logico e, superandoli, pone le basi per una filosofia capace di recuperare il significato del problema di Dio, un Dio però - non va dimenticato - di cui il linguaggio non può parlare, ma solo il vissuto può mostrare.

Risultati analoghi a quelli di Wittgenstein saranno ottenuti, in discipline adiacenti, con la dimostrazione: dei teoremi di incompletezza dei sistemi assiomatici (Gödel 1931); della necessità di metalinguaggi e impossibilità di una definizione di tutti gli enunciati veri di un sistema (Tarski 1935); dei limiti di ogni operazione logica automatizzata e incapacità di "giudicare dal di fuori” il processo (Turing 1937); dell'esistenza di nozioni di infinito che non appartengono alla matematica (Cantor 1884). Tutti questi autori coincidono nell'affermare che la semantica del linguaggio scientifico non è esaurita dal formalismo delle scienze empiriche: esiste uno spazio "significativo" per nozioni che appartengano ad un meta-linguaggio, ovvero trascendano l'analisi empirica.

\section{Il carattere ontologicamente incompiuto della realtà fisica contingente e la sua apertura verso un fondamento metafisico-necessario}

L'impraticabilità sul piano logico di una scienza autoreferenziale possiede un suo corrispettivo anche sul piano ontologico. Come l'analisi delle scienze non può definire in modo completo, chiuso a dimensioni meta-empiri- 
che, il linguaggio scientifico e i criteri di verità di cui esso ha bisogno, così essa necessita anche di pre-supposti di carattere ontologico. Previamente all'analisi delle scienze, occorre cioè che gli enti materiali "esistano" ed "esistano secondo una specifica natura".

La necessità di un implicito fondamento ontologico per l'attività delle scienze, può essere messa in luce sviluppando le seguenti prospettive:

a) alla base di ogni scienza naturale si trova una filosofia della natura ed alla base di ogni filosofia della natura esiste un'ontologia: tuttavia, ogni ontologia deve affrontare il problema della contingenza dell'essere

b) affinché la scienza possa studiare i suoi oggetti, occorre che questi esistano (come enti): la scienza non può dare ragione della loro esistenza, nemmeno del perché ultimo dell'essere in quanto tale, occupandosi soltanto delle trasformazioni tra gli enti;

c) l'analisi delle scienze naturali poggia sulla specificità formale (essenza, natura) delle cose, insieme alla loro esistenza;

L’analisi empírica richiede perciò due presupposti: gli enti materiali esistono, ed esistono secondo una natura specifica. Le nozioni di "essere" e di "essenza/natura", proprie della filosofia della natura, sono presupposti indeducibili dall'interno del metodo delle scienze naturali, che rendono tuttavia la scienza possibile. Riconoscere l'esistenza di tali presupposti ontologici equivale a riconoscere un'area semantica di intelligibilità che trascende il reale fisico-empirico.

La negazione dell'esistenza di un'area semantica che "trascenda" l'analisi empirica delle scienze è propria del tentativo di fondare una scienza autoreferenziale, che eluda il problema dell'essere, ignorandolo (materialismo) o volendone dare giustificazione all'interno del formalismo empirico.

Ciò genera alcune inevitabili contraddizioni, specialmente nel campo della cosmologia fisica, quando questa nega l'esistenza di un fondamento ontologico che sostiene la sua analisi sul reale fisico. Ad esempio, se la cosmologia termina attribuendo alla materia le proprietà di un Assoluto filosofico (eternità, necessità, prima causa incausata, ecc.), nonostante l'esperienza della sua contingenza; oppure se attribuisce ad un formalismo 
matematico coerente, di carattere descrittivo, il valore di ragione sufficiente per spiegare l'esistenza del cosmo che esso descrive o rappresenta; o, ancora, quando cerca di dare ragione dell'esistenza e della natura del tempo dall'interno del tempo stesso; o, infine, se tenta di "dare ragione dell'intero", cercando una legge o una formulazione totalizzante, che risulti fondativa per tutto il reale ("teoria del tutto" - Theory of Everything), incontrando le medesime incompletezze e antinomie che sorgono in ambito logico.

In sostanza, la ricerca scientifica percepisce la necessità di dover ammettere un fondamento esterno al suo metodo quando riconosce che l'analisi del reale fisico prende sempre avvio da qualche grandezza misurabile, implicitamente assunta (massa, topologia, spazio-tempo, vuoto fisico, energia virtuale, ecc.), a partire dalla quale è possibile costruire le successive probabilità di esistenza di enti da essa derivati.

Se la teoria della supergravità raggiunge l'obiettivo che si propone, ci dirà non solo perché ci sono le particelle che esistono, e non altre, ma anche perché hanno la massa, la carica e le altre proprietà che le contraddistinguono. Tutto ciò potrebbe derivare da una teoria matematica che raccogliesse tutta la fisica (in senso riduzionista) in un'unica superlegge.

Ma si propone di nuovo l'interrogativo: perché quella superlegge, e non un'altra? È questo l'interrogativo ultimo, terminale: la fisica potrà forse spiegare il contenuto, l'origine e l'organizzazione dell'universo fisico, ma non le leggi (o la superlegge) della fisica stessa. Tradizionalmente si attribuisce a Dio l'invenzione delle leggi di natura e la creazione delle cose (lo spazio-tempo, gli atomi, gli uomini e tutto il resto) su cui tali leggi si applicano. Nello scenario del "pasto gratuito" bastano le leggi soltanto: l'universo al resto può provvedere da sé, la propria creazione compresa. Ma, e le leggi? Occorre che già esistessero, le leggi, in modo che l'universo potesse esistere. Bisogna in un certo senso che la fisica quantistica esista affinché una transizione quantica possa generare il cosmo (Davies 2002, 298).

L'esistenza di un fondamento ontologico che dia ragione dell'essere e della specifica essenza degli enti materiali, che sia anche la causa/ragione ultima 
della presenza di forma/informazione che trascende la materia stessa, rimanda ad un'area semantica di intelligibilità che la scienza "non considera un nonsenso", e la cui esistenza "coglie come ragionevole".

Chiamiamo allora con il nome generico di "discorso sul Logos" un discorso che possa svilupparsi entro tale area semantica di intelligibilità, colta nei due precedenti passi, che corrispondono anche a due "finestre" del metodo scientifico verso l'esterno, cioè come necessità di un trascendimento del linguaggio formale e come necessità di un fondamento ontologico che dia ragione di ogni rappresentazione empirica.

Nell'attività di ricerca dello scienziato, ovvero nelle riflessioni filosofiche che egli realizza a partire da tale attività, un certo rimando ad un Logos pare cogliersi quando: a) egli vede nella natura un'alterità "oggettivo-razionale"; e quando: b) lo studio del reale lo pone in rapporto con un'alterità "soggettivo-dialogica". Vediamo nelle prossime due sezioni entrambi questi aspetti.

\section{La percezione di un Logos ut ratio nell'analisi delle scienze naturali}

Uno dei modi con cui lo scienziato accede alla nozione di Logos è la riflessione sul motivo della razionalità dell'universo, sulle ragioni dell'intelligibilità e della stabilità delle leggi di natura. Il reale fisico, infatti, può essere compreso in termini matematici; si presenta con leggi stabili nel tempo e nello spazio; in esso le stesse particelle elementari sono tutte rigorosamente identiche e le proprietà fisico-chimiche dei vari elementi seguono precise strutture di ordinamento.

L'universo fisico manifesta una sorta di "fondamento di razionalità" col quale il ricercatore viene inevitabilmente in contatto. Segnalato per primo da Maxwell, l'“enigma” dell'intelligibilità fu discusso da Planck, de Broglie, Einstein; in tempi più recenti, fra gli altri, è stato menzionato da Paul Davies, John Barrow e Roger Penrose. Lo testimoniano, fra le molte possibili, le seguenti affermazioni: 
Noi non ci meravigliamo abbastanza del fatto che una scienza sia possibile, cioè che la nostra ragione ci fornisca i mezzi per comprendere almeno certi aspetti di ciò che accade intorno a noi nella natura (de Broglie 1950, 216).

Si potrebbe dire che l'eterno mistero del mondo è la sua comprensibilità. Il fatto che sia comprensibile è davvero un miracolo (Einstein 1936/1988, 530).

Lei trova strano che io consideri la comprensibilità della natura (per quanto siamo autorizzati a parlare di comprensibilità), come un miracolo o un eterno mistero. Ebbene, ciò che ci dovremmo aspettare, a priori, è proprio un mondo caotico del tutto inaccessibile al pensiero. Ci si potrebbe (di più, ci si dovrebbe) aspettare che il mondo sia governato da leggi soltanto nella misura in cui interveniamo con la nostra intelligenza ordinatrice: sarebbe un ordine simile a quello alfabetico, del dizionario, laddove il tipo d'ordine creato ad esempio dalla teoria della gravitazione di Newton ha tutt'altro carattere. Anche se gli assiomi della teoria sono imposti dall'uomo, il successo di una tale costruzione presuppone un alto grado d'ordine del mondo oggettivo, e cioè un qualcosa che, a priori, non si è per nulla autorizzati ad attendersi (Einstein 1952/1988, 740).

L'osservazione dell'ordine e della razionalità del cosmo viene messa talvolta in diretta relazione con la nozione di Dio, come in queste due pagine di Albert Einstein e Paul Davies:

È certo che alla base di ogni lavoro scientifico un po' delicato si trova la convinzione, analoga al sentimento religioso, che il mondo è fondato sulla ragione e può essere compreso. Questa convinzione legata al sentimento profondo della esistenza di una mente superiore che si manifesta nel mondo della esperienza, costituisce per me l'idea di Dio (Einstein 1988, 32).

Attraverso il mio lavoro scientifico sono giunto a credere sempre più fermamente che l'universo fisico è costruito con un'ingegnosità così sorprendente che non riesco a considerarlo meramente come un fatto puro e semplice. Mi pare che ci debba essere un livello più profondo di spiegazione. Se si desidera chiamare tale livello Dio è una questione di gusto e di definizione (Davies 1993, 7).

Il metodo scientifico non può dimostrare, se la razionalità, l'intelligibilità o l'ordine rispondano ad un “disegno progettuale”. Di fatto, le scienze empiriche non possono inferire l'esistenza di una causalità finale di tipo intenzionale, ma possono mettere in luce solo i livelli inferiori di tale fina- 
lismo, cogliendolo come "coerenza" e "razionalità", o anche come "teleonomia” (specie in ambito biologico). Di conseguenza, il Logos che la scienza coglie spesso non rimanda al di là delle leggi stesse. Ciò spiega il frequente ricorso ad espressioni come "codice cosmico", "cosmo intelligente", "mente cosmica”, "teoria del tutto" (intesa come legge universale immanente). Dal nostro punto di vista le cose non cambiano molto perché, in entrambi i casi, si sta accedendo ad un'area semantica riconosciuta significativa, che rimanda al di là del metodo scientifico in senso stretto. In alcuni autori, come ad esempio Freeman Dyson, la percezione della razionalità non rimanda ad una nozione di Dio, ma ad una semplice Intelligenza.

Dall'esistenza di queste coincidenze fisiche ed astronomiche ricavo la conclusione che l'universo è un luogo straordinariamente ospitale, come possibile habitat di creature viventi. E poiché sono uno scienziato abituato ai modi di pensiero e al linguaggio del ventesimo secolo, e non a quello del diciottesimo, non affermo che l'architettura dell'universo dimostra l'esistenza di Dio. Affermo soltanto che l'architettura dell'universo è coerente con l'ipotesi che la mente abbia un ruolo essenziale nel suo funzionamento (Dyson 1979, 290-291).

La posizione implicita più frequente diviene allora quella panteista, dove l'universo e l'Intelligenza che lo governa sono la stessa cosa, coincidono. Lo scienziato può superare la posizione panteista solo con una successiva astrazione, mettendo ad esempio in luce il problema della contingenza: se il cosmo ha una mente che non lo trascende, tale mente sarà contingente come il cosmo stesso; sarebbe più sensato ipotizzare l'esistenza di un Assoluto che desse ragione sia della razionalità della realtà fisica, sia dell'esistenza di un fondamento necessario in sé, e che si proponga, pertanto, non come "uno col mondo", bensì come "Altro dal mondo". A sostegno del superamento del panteismo viene incontro anche il senso illativo, che pone la percezione della razionalità del cosmo in rapporto con altre forme di conoscenza che suggeriscono, o in alcuni casi perfino filosoficamente garantiscono, un accesso ad un Assoluto trascendente.

Resta tuttavia assai importante che la domanda sul motivo della razionalità e dell'ordine emerga dall'interno delle scienze "in quanto domanda". 
Interrogativi quali: perché l'universo è razionale?, perché le sue leggi sono intelligibili?, perché esiste una sintonia fra la struttura del cosmo e le leggi che rendono possibile la vita?, ecc., sono questioni che puntano verso un'area di senso, verso un Logos che le scienze riconoscono significativo.

\section{La percezione di un Logos ut verbum nell'attività di ricerca dello scienziato}

Lo scienziato è protagonista di un'altra importante percezione: egli coglie nella realtà fisica una sorta di "alterità dialogica", si sorprende della sua capacità di "dialogare" con la natura e si chiede quanto ciò sia significativo. L'attività scientifica può assomigliare in alcuni casi ad un dialogo fra l'uomo e l'Assoluto.

[lo scienziato] prende coscienza dell'ordine centrale [del mondo] con la stessa intensità con cui si entra in contatto con l'anima di un'altra persona (Heisenberg 1984, 225).

I fisici padroneggiano faticosamente le tecniche matematiche perché l'esperienza ha insegnato loro che esse costituiscono la via migliore, anzi l'unica, per capire il mondo fisico. Scegliamo quel linguaggio perché è l'unico col quale il cosmo ci parla (Polkinghorne 1987, 72).

Nella descrizione della fenomenologia del lavoro scientifico si nota non di rado l'impiego del termine "rivelazione".

Quando l'attività scientifica accede alla realtà cogliendovi l'esistenza di un'alterità dialogica, la natura viene allora riconosciuta come meritevole di essere studiata, capace di motivarne il corrispondente sforzo intellettuale, perché capace di legare ad una verità ed una bellezza indipendenti dal soggetto conoscente. La ricerca scientifica non è colta come mero impegno verso se stessi o verso la comunità scientifica, bensì come impegno verso la verità, alla quale ci si dirige con passione intellettuale. Come già segnalato da alcuni autori, sia la ricerca della verità, propria di ogni autentica indagine scientifica, sia l' "esperienza dei fondamenti”, percepita di fronte alle incompletezze di ordine logico e ontologico, possono confluire in un accesso all'Assoluto, e dunque in una esperienza di natura religiosa. 
Nei più grandi scienziati l'esperienza scientifica della verità è in qualche senso theoria, cioè una visione di Dio (von Laue 1950, 4).

Nessun poeta e profeta ha contemplato prodigi così profondi come quelli che si rivelano allo scienziato. Pochi saranno così ottusi da non reagire alla conoscenza materiale di questo nostro mondo con un senso di timore reverenziale che merita di essere definito religioso (Simpson 1972, 213).

Segnalava la disponibilità dell'esperienza scientifica a fornire linfa ad una vera esperienza del "religioso” anche Mariano Artigas, in una pagina della sua già citata opera The Mind of the Universe: «A religious attitude basically implies openness toward God and a new outlook that stems from the contemplation of the divine dimensions of the world and every one of its parts, especially other human beings. Insofar as scientific progress favors this outlook, it can be considered a source of religious inspiration» (Artigas 2000, 331).

Nella considerazione dell'esperienza scientifica come percezione del sacro, è vero, sono possibili anche delle ambiguità. L'immagine dell'Assoluto percepita dalla razionalità scientifica è espressa con un linguaggio filosoficamente impreciso, sovente mescolato ad ambiguità e non poche volte colorato di panteismo o di deismo. Non sfugge la difficoltà, già nota in sede filosofica generale, di poter cogliere il carattere personale dell'Assoluto. Sarebbe però un errore, a nostro avviso, non valorizzare questo accesso, eppure impreciso e sfuocato. Il filosofo e il teologo, piuttosto che negarlo e metterne in luce la debolezza, dovrebbero purificarlo e rafforzarlo, mostrandone la vera immagine, quella di un Assoluto che trascende l'universo perché distinto da esso.

In realtà, più “personalistici” sono i canoni con cui lo scienziato si pone di fronte al mondo reale, meno profonda si fa tale distanza. Se un approccio impersonale appare infatti in maggior sintonia con il panteismo (All is One), chi non perde mai di vista la propria personalità di fronte al mondo e condivide una conoscenza realista delle cose, sarà più facilmente disposto ad ascoltare la natura, a stupirsi di fronte ad essa, a riconoscerla dunque come altra-da-sé e, il suo Creatore, Altro dalla natura e altro da sé stessi. 
Su questa medesima linea, l'idea di poter cogliere il Logos in quanto Verbum fa sì che si possa parlare - come di fatto avviene nelle testimonianze di alcuni scienziati - anche di "rivelazione", con la quale la natura si fa incontro al ricercatore. Dove questa si manifesta, essa non è più qualificabile come semplice "intuizione", comprensione immediata di qualcosa che era oscuro e adesso si vede con chiarezza, visione di una coerenza che in definitiva potrebbe avere solo un'origine psicologica. Le dichiarazioni di molti uomini di scienza sembrerebbero voler dire qualcosa di più: si tratta piuttosto di una parola (logos) esterna al soggetto, che esercita su di lui un appello, un messaggio che suscita sorpresa e muove implicitamente al rispetto, alla gratitudine e talvolta anche alla lode, come forma implicita di dialogo. Questo Logos è legato ad un'esperienza estetica, viene segnalato con i caratteri di un mistero che contiene il senso nascosto del mondo, ed ha come riferimento gnoseologico una metafisica implicita, aperta sul reale e disposta ad imparare dalla natura e dalle sue leggi. A quel punto diviene più facile ammettere che non è la natura, di per sé, a rivelare alcunché, ma è Qualcuno che si rivela attraverso di essa.

\section{Osservazioni conclusive}

Al termine di questo percorso, possiamo rispondere alla domanda postaci in partenza, e cioè se esista un discorso su Dio intelligibile anche per la razionalità scientifica del nostro tempo. Abbiamo visto che esiste un'area semantica disponibile per un discorso sull'Assoluto, un'area che la razionalità scientifica può riconoscere, ovvero indicare, come qualcosa di significativo anche per chi, come lo scienziato, studia la natura con i metodi e gli strumenti propri del lavoro sperimentale e del formalismo logico-matematico. In particolare, si può accedere a quest'area attraverso quattro "finestre", che corrispondono ad altrettanti "trascendimenti", ma anche a dei veri e propri "fondamenti" della scienza. Sono tali i fondamenti ontologici e logici dell'analisi empirica, evidenziati dalle incompletezze, e dunque dalle sue aperture, ontologiche (verso l'essere) ed epistemologiche (verso le nozioni di natura, forma, verità, ecc.). Sono tali le aperture verso la percezione 
di un Logos, colto come ragione intelligibile, ma anche come sorgente di senso e di motivazione che spinge il ricercatore a considerare la natura meritevole di essere studiata e la verità meritevole di essere cercata.

In sostanza, possiamo proporre le seguenti osservazioni conclusive:

a) Il reale fisico si "ostende" a noi con una sua "datità" (givenness) che la scienza non crea, ma riceve; ciò che si impone all'esperienza scientifica come qualcosa di dato, può dare origine ad un'esperienza religiosa che "riconosce il dato come donato" e sa cogliere un passaggio dalla percezione di un logos ut ratio a quella di un logos ut verbum.

b) Nel descrivere la loro esperienza di studio e di ricerca, molti uomini di scienza parlano del reale fisico come di un'" alterità oggettiva e coerente, caratterizzata da una specificità formale”. Il collegamento fra questa percezione e la nozione di Assoluto può avvenire operando un passaggio dal "problema” dei fondamenti ad un'“esperienza" dei fondamenti, che rende la ricerca scientifica simile ad una "esperienza del sacro".

c) Lo studio scientifico della natura continua a manifestare una sua apertura al "mistero" e resta ragionevole chiedersi se il mondo abbia una spiegazione; la ricerca di questa spiegazione rimanda ad una nozione o ad un'area semantica che non è considerata un nonsenso, ove acquista significato la possibilità di un logos su Dio.

Dunque quest'area sembra esistere, ed esiste semplicemente perché il metodo e gli strumenti del lavoro scientifico possono essere trascesi senza essere contraddetti. Qui giace la possibilità di una nozione di "Dio" significativa anche per l'uomo di scienza, una nozione che non venga da lui giudicata come un "non-senso", ma continui a fare appello alla sua attività scientifica e alla sua vita.

\section{Bibliografia}

Artigas, Mariano. 2000. The Mind of the Universe. Radnor: Templeton Foundation Press.

Davies, Paul C.W. 1993. La mente di Dio. Milano: Mondadori.

Davies, Paul C.W. 2002. Dio e la nuova fisica. Milano: Mondadori. 
De Broglie, Louis. 1950. Fisica e Metafisica. Torino: Einaudi.

Dyson, Freeman. 1979. Turbare l'universo. Torino: Boringhieri.

Einstein, Albert. 1936/1988. “Fisica e realtà.” In Opere scelte. Torino: Bollati-Boringhieri.

Einstein, Albert. 1952/1988. “Lettera a M. Solvine (30.3.1952).” In Opere scelte. Torino: Bollati-Boringhieri.

Einstein, Albert. 1988. Come io vedo il mondo. Roma: Newton Compton.

Heisenberg, Werner. 1984. Fisica e oltre. Torino: Boringhieri.

Polkinghorne, John. 1987. Scienza e fede. Milano: Mondadori.

Simpson, George. 1972. Evoluzione. Una visione del mondo. Firenze.

Tommaso d'Aquino. Summa theologiae.

Von Laue, Max. 1950. History of Physics. New York: Academic Press.

Wittgenstein, Ludwig. 1922. Tractatus Logico-philosophicus. New York and London: Harcourt and Kegan Paul. 